\title{
Association of diabetes with atrial fibrillation types: a systematic review and meta-analysis
}

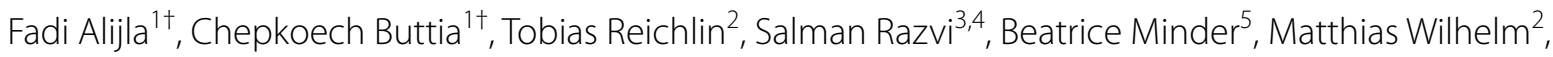
Taulant Muka', Oscar H. Franco ${ }^{1}$ and Arjola Bano ${ }^{1,2^{*}}$

\begin{abstract}
Background: Atrial fibrillation (AF) is a common arrhythmia classified as paroxysmal and non-paroxysmal. Non-paroxysmal AF is associated with an increased risk of complications. Diabetes contributes to AF initiation, yet its role in AF maintenance is unclear. We conducted a systematic review and meta-analysis to summarize the evidence regarding the association of diabetes with AF types.
\end{abstract}

Methods: We searched 5 databases for observational studies investigating the association of diabetes with the likelihood of an AF type (vs another type) in humans. Study quality was evaluated using the Newcastle-Ottawa Scale. Studies classifying AF types as paroxysmal (reference) and non-paroxysmal were pooled in a meta-analysis using random effects models.

Results: Of 1997 articles we identified, 20 were included in our systematic review. The population sample size ranged from 64 to 9816 participants with mean age ranging from 40 to 75 years and percentage of women from 24.8 to $100 \%$. The quality of studies varied from poor (60\%) to fair (5\%) to good (35\%). In the systematic review, 8 studies among patients with AF investigated the cross-sectional association of diabetes with non-paroxysmal AF (vs paroxysmal) of which 6 showed a positive association and 2 showed no association. Fourteen studies investigated the longitudinal association of diabetes with "more sustained" AF types (vs "less sustained") of which 2 showed a positive association and 12 showed no association. In the meta-analysis of cross-sectional studies, patients with AF and diabetes were 1.31-times more likely to have non-paroxysmal AF than those without diabetes [8 studies; pooled OR (95\% Cl), 1.31 (1.13-1.51), I' $=82.6 \%$. The meta-analysis of longitudinal studies showed that for patients with paroxysmal AF, diabetes is associated with 1.32-times increased likelihood of progression to non-paroxysmal AF [five studies; pooled OR (95\% Cl), $\left.1.32(1.07-1.62) ;\left.\right|^{2}=0 \%\right]$.

Conclusions: Our findings suggest that diabetes is associated with an increased likelihood of non-paroxysmal AF rather than paroxysmal AF. However, further high quality studies are needed to replicate these findings, adjust for potential confounders, elucidate mechanisms linking diabetes to non-paroxysmal AF, and assess the impact of antidiabetic medications on AF types. These strategies could eventually help decrease the risk of non-paroxysmal AF among patients with diabetes.

Keywords: Diabetes mellitus, Paroxysmal, Non-paroxysmal, Persistent, Permanent, Atrial fibrillation

*Correspondence: arjola.bano@ispm.unibe.ch

${ }^{\dagger}$ Fadi Alijla and Chepkoech Buttia contributed equally to this work

${ }^{1}$ Institute of Social and Preventive Medicine (ISPM), University of Bern, Mittelstrasse 43, 3012 Bern, Switzerland

Full list of author information is available at the end of the article

\section{Introduction}

Atrial fibrillation (AF) is the most common arrhythmia, and it is associated with high risk of morbidity and mortality [1-4]. The incidence and prevalence of AF are increasing worldwide [5]. According to recent guidelines, $\mathrm{AF}$ is classified into the following types: first diagnosed,

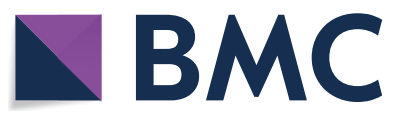

(c) The Author(s) 2021. Open Access This article is licensed under a Creative Commons Attribution 4.0 International License, which permits use, sharing, adaptation, distribution and reproduction in any medium or format, as long as you give appropriate credit to the original author(s) and the source, provide a link to the Creative Commons licence, and indicate if changes were made. The images or other third party material in this article are included in the article's Creative Commons licence, unless indicated otherwise in a credit line to the material. If material is not included in the article's Creative Commons licence and your intended use is not permitted by statutory regulation or exceeds the permitted use, you will need to obtain permission directly from the copyright holder. To view a copy of this licence, visit http://creativecommons.org/licenses/by/4.0/. The Creative Commons Public Domain Dedication waiver (http://creativeco mmons.org/publicdomain/zero/1.0/) applies to the data made available in this article, unless otherwise stated in a credit line to the data. 
paroxysmal, persistent, long-standing persistent, and permanent AF (Additional file 1: Table S1) [6]. While paroxysmal AF terminates within 7 days of onset, nonparoxysmal AF (including persistent, long-standing persistent, and permanent AF) lasts longer [6]. Patients who develop non-paroxysmal AF have a higher risk of adverse events (including all-cause mortality, thromboembolism, and hospitalization) compared to those with paroxysmal AF $[7,8]$. Therefore, the identification of modifiable risk factors for non-paroxysmal AF is important to improve pathophysiological understandings about AF development and to improve clinical management and prognosis for patients with AF by avoiding AF progression to nonparoxysmal AF.

Diabetes mellitus represents a major risk factor for AF [9]. Patients with diabetes have a $34 \%$ greater risk of AF compared to those without diabetes [10]. Several mechanisms, such as electrical, structural, and autonomic atrial remodelling, can explain the role of diabetes in the initiation of AF [11]. However, the impact of diabetes on the maintenance of sustained AF in non-paroxysmal AF remains unelucidated. To date, epidemiological studies have investigated the association of diabetes with different classifications of AF types [12-15]. Various forms of "more sustained AF" (i.e., non-paroxysmal AF, permanent $\mathrm{AF}, \mathrm{AF}$ progression of paroxysmal to non-paroxysmal AF, and persistent to permanent $\mathrm{AF}$ ) have been compared with forms of "less sustained AF." However, results across studies have been inconsistent. For example, one study reported that among patients diagnosed with $\mathrm{AF}$, those with and without diabetes have significant differences in AF types [13], while another study found no differences [16]. A study including patients with paroxysmal AF reported an association between diabetes and the development of permanent AF [14], yet another study found no association [12]. These inconsistencies may also arise from differences in definitions of AF types, differences in characteristics of participants, or small study sample sizes. Overall, it is unclear whether patients with and without diabetes have differences in AF types.

We therefore performed a systematic review, which aimed to summarize evidence regarding the association of diabetes with the likelihood of having a certain AF type, rather than another AF type. In order to provide insights concerning the role of diabetes on AF's pathophysiology, we also performed a meta-analysis of diabetes' association with the likelihood of having nonparoxysmal AF rather than paroxysmal AF.

\section{Methods}

\section{Data sources and search strategy}

We conducted our systematic review following a recently published guide about performing systematic reviews and meta-analyses [17]. The guide was based on the Preferred Reporting Items for Systematic Review and Metaanalyses recommendations [18]. We searched Embase, Medline Ovid, Cochrane Central, Web of Science Core Collection, and Google Scholar from inception until 1 October 2021 [18]. To identify relevant articles, we combined (a) diabetes-related terms with (b) AF-related terms, such as "atrial," "fibrillation," "paroxysmal," "persistent," "permanent," and (c) article type. We excluded conference abstracts, letters to the editor, and editorials. (Additional file 1: Appendix A). We performed our search with the assistance of an experienced information specialist. We used EndNote to manage references.

\section{Study selection}

Eligibility criteria for included studies required (1) observational studies, including cross-sectional, prospective, case-control, nested case-control, or nested case-cohort studies of humans that (2) investigated the association of diabetes with the likelihood of having a certain AF type rather than another AF type and (3) provided information about effect estimates (e.g., risk ratios, hazard ratios [HRs], and odds ratios [ORs]) with 95\% confidence intervals [95\% CIs] or p-values). We excluded animal studies, reviews, meta-analyses, conference abstracts, conference proceedings, poster presentations, case-series, and letters to the editor. We did not restrict publication year or language.

Two reviewers independently screened eligible citations by title and abstract. Furthermore, these two reviewers independently evaluated full texts of eligible articles. In cases of disagreement between reviewers, decisions were either made by consensus or in consultation with a third reviewer. We hand searched the reference lists of included articles to identify additional studies.

\section{Data extraction}

Two authors independently extracted the following data from each study: first author's last name, year of publication, country where the study was conducted, study population, number of participants at baseline, mean age, percentage of women, diabetes assessment, adjustments for potential confounders, outcome, follow-up time, and risk estimates with $95 \%$ CIs.

\section{Quality assessment}

Two authors independently performed quality assessment of the included studies using the NewcastleOttawa Scale (NOS) [19]. NOS rates study quality from 0 to 9 stars according to three domains: selection, comparability, and outcome assessment. Based on thresholds for converting NOS scores into Agency for Healthcare 
Research and Quality standards, we categorized study quality as (I) good (selection: 3-4 stars; comparability: 1-2; outcome assessment: 2-3); (II) fair (selection: 2; comparability: 1-2; outcome assessment: $2-3$ ); or (III) poor (selection: 0-1; comparability: 0; outcome assessment: 0-1) (Additional file 1: Appendix B) [19, 20].

\section{Statistical analyses}

We included eligible studies that classified AF types into paroxysmal (reference) and non-paroxysmal AF in the meta-analysis. When applicable, ORs and 95\% CIs were calculated using 2 by 2 tables. Effect estimates on the association of diabetes with the likelihood of non-paroxysmal AF (vs paroxysmal AF) were pooled using random effects models as described by DerSimonian and Laird; and forest plots were constructed [21]. We assessed heterogeneity using the $\mathrm{I}^{2}$ statistic; we considered $\mathrm{I}^{2} \leq 25 \%$ low; $25 \%<\mathrm{I}^{2}<75 \%$ moderate; and $\mathrm{I}^{2} \geq 75 \%$ high. In order to test the robustness of our results, we performed several sensitivity analyses: (1) we recalculated estimates after removing studies one by one from the pooled analysis to evaluate the role of individual studies on the overall results; (2) we performed subgroup analyses based on follow-up time (i.e., $\leq 1$ year vs $>1$ year) in our metaanalysis of longitudinal studies; and (3) we also restricted our meta-analysis of longitudinal studies to studies that defined AF types in accordance with the guidelines [6]. We used funnel plots and Egger regression symmetry tests to evaluate the possibility of publication bias [22]. Statistical analyses were performed using Stata IC version 15.1 (StataCorp LLC, Texas, USA).

\section{Results}

\section{Literature search}

After excluding duplicates, we identified 1,997 relevant citations. We further screened studies by title and abstract, identifying 33 potentially relevant articles. After examining the article full texts, we identified 17 unique eligible articles. Our reference search yielded 3 additional articles, resulting in a total of 20 articles that we included in our systematic review. We present the results of our search strategy in Additional file 1: Fig. S1.

\section{Characteristics of included studies}

The population sample size ranged from 64 to 9816 participants. The mean age ranged from 40 to 75 years. The percentage of women ranged from 24.8 to $100 \%$. We present the general characteristics of the 20 eligible articles in Tables 1 and 2 and Additional file 1: Table S2. Of the 20 included studies, 5 were conducted in the United States [12, 23-26], 8 in Europe [7, 13-16, 27-29], 3 in Japan [30-32], 1 in Canada [33], 1 in Australia [34], and 2 across several continents [35, 36]. Four studies diagnosed diabetes based on medical files and/or use of antidiabetic treatment [12, 23, 24, 32] (Tables 1 and 2). Sixteen studies did not provide information about assessment of diabetes [7, 13-16, 25-31, 33-37] (Tables 1 and 2). We provide detailed information about the studies' recruitment settings and periods, inclusion and exclusion criteria, AF monitoring, and AF definitions across studies in Additional file 1: Tables S2, S3 and S4.

\section{Cross-sectional association of diabetes with AF types}

Our systematic review included 8 studies performed among patients with AF investigating the cross-sectional association of diabetes with the likelihood of having nonparoxysmal AF rather than paroxysmal AF $[13,15,16,23$, $27,28,34,36$ ] (Table 1). Of the eight studies, six studies reported unadjusted estimates [13, 15, 23, 27, 28, 36], and two studies reported adjusted and unadjusted estimates $[16,34]$ (Table 1). Of the eight studies, six studies showed an association of diabetes with an increased likelihood of non-paroxysmal AF (with ORs varying from 1.2 to 1.8 ) $[13,23,27,28,34,36]$; and 2 studies $[15,16]$ showed no association. We included these 8 studies in the metaanalysis since all eight studies classified AF types into paroxysmal (reference) vs non-paroxysmal. The metaanalysis showed that diabetes is associated with a 1.31times increased likelihood of having non-paroxysmal AF rather than paroxysmal AF with high heterogeneity (pooled OR [95\% CI], 1.31 [1.13-1.51], $\mathrm{I}^{2}=82.6 \%$ ) (Fig. 1). All studies we included in our meta-analysis provided unadjusted estimates. Results remained consistent when pooling the two studies reporting adjusted estimates (including adjustments for age, sex, smoking, physical activity, cardiovascular risk factors, and cardiovascular diseases) with the six studies reporting unadjusted estimates (pooled OR [95\% CI], 1.29 [1.11-1.50], $\left.\mathrm{I}^{2}=82.5 \%\right)$.

\section{Longitudinal association of diabetes with AF types}

Our systematic review included 14 studies investigating the longitudinal association of diabetes with AF types $[7,12,14,15,23-26,29-33,35]$ (Table 2). Of the 14 studies, six studies reported unadjusted estimates [7, 26, 31-33, 35], six studies reported adjusted estimates [12, 14, 24, 25, 29, 30], and 2 studies reported adjusted and unadjusted estimates [15, 23] (Table 2). One study was performed among patients without AF at baseline; it showed no association between diabetes and the likelihood of developing non-paroxysmal AF rather than paroxysmal AF [25]. Five studies were performed among patients with paroxysmal or persistent AF at baseline; they showed no association between diabetes and the likelihood of developing permanent $\mathrm{AF}[24,30]$ or "AF progression" (i.e., paroxysmal to 


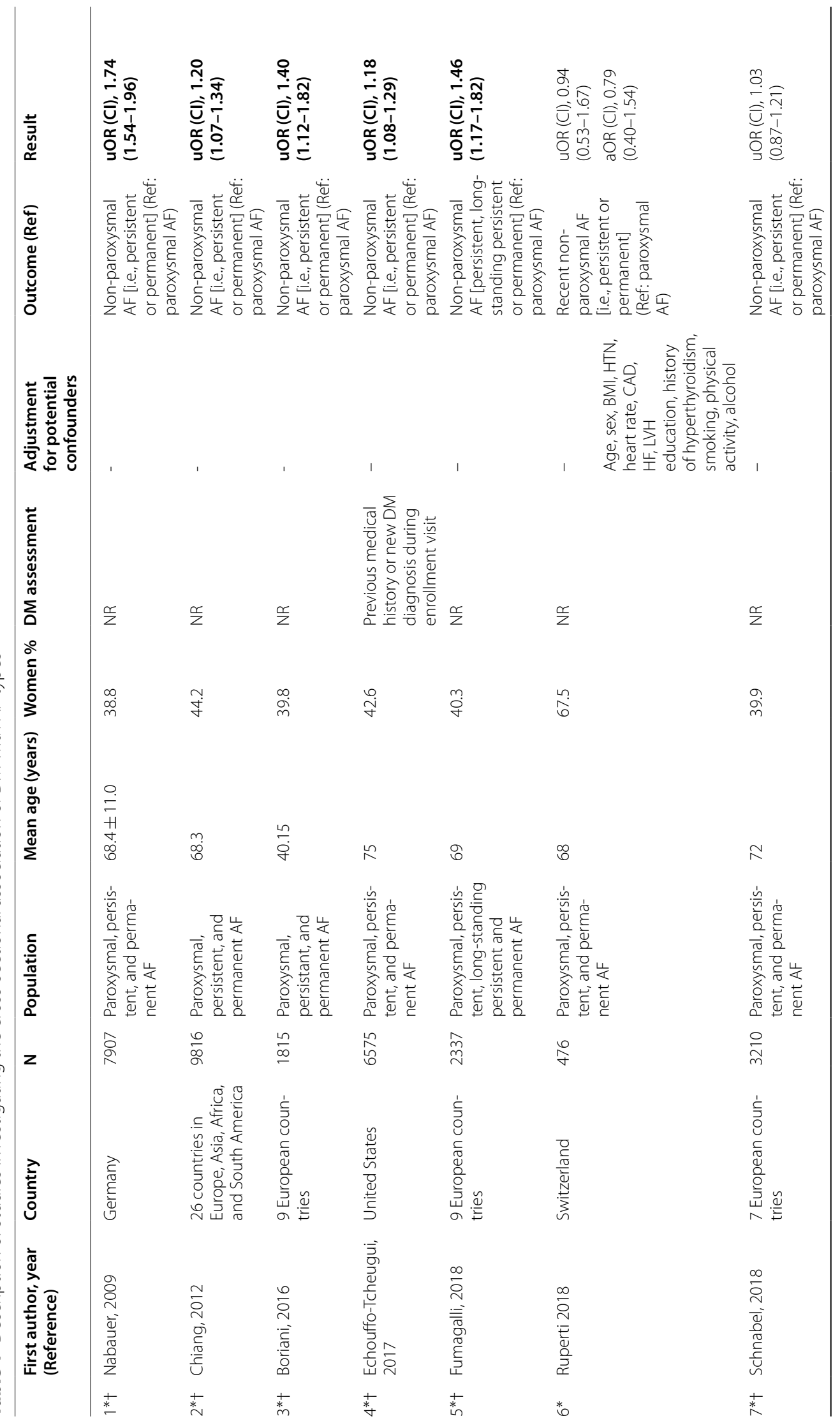




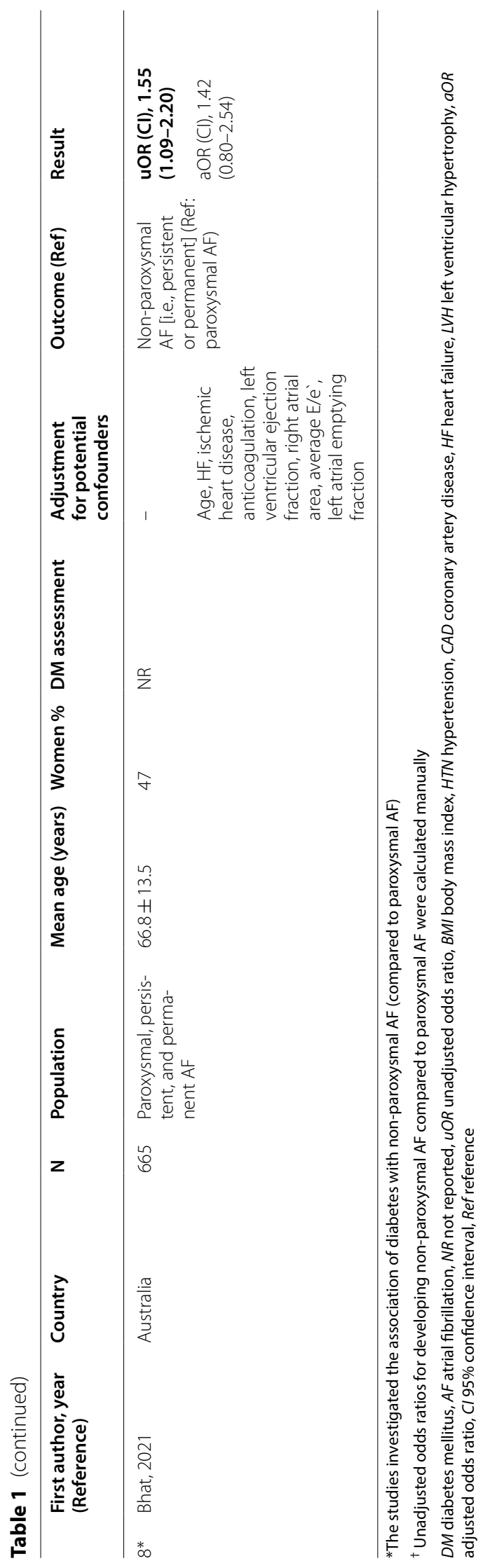




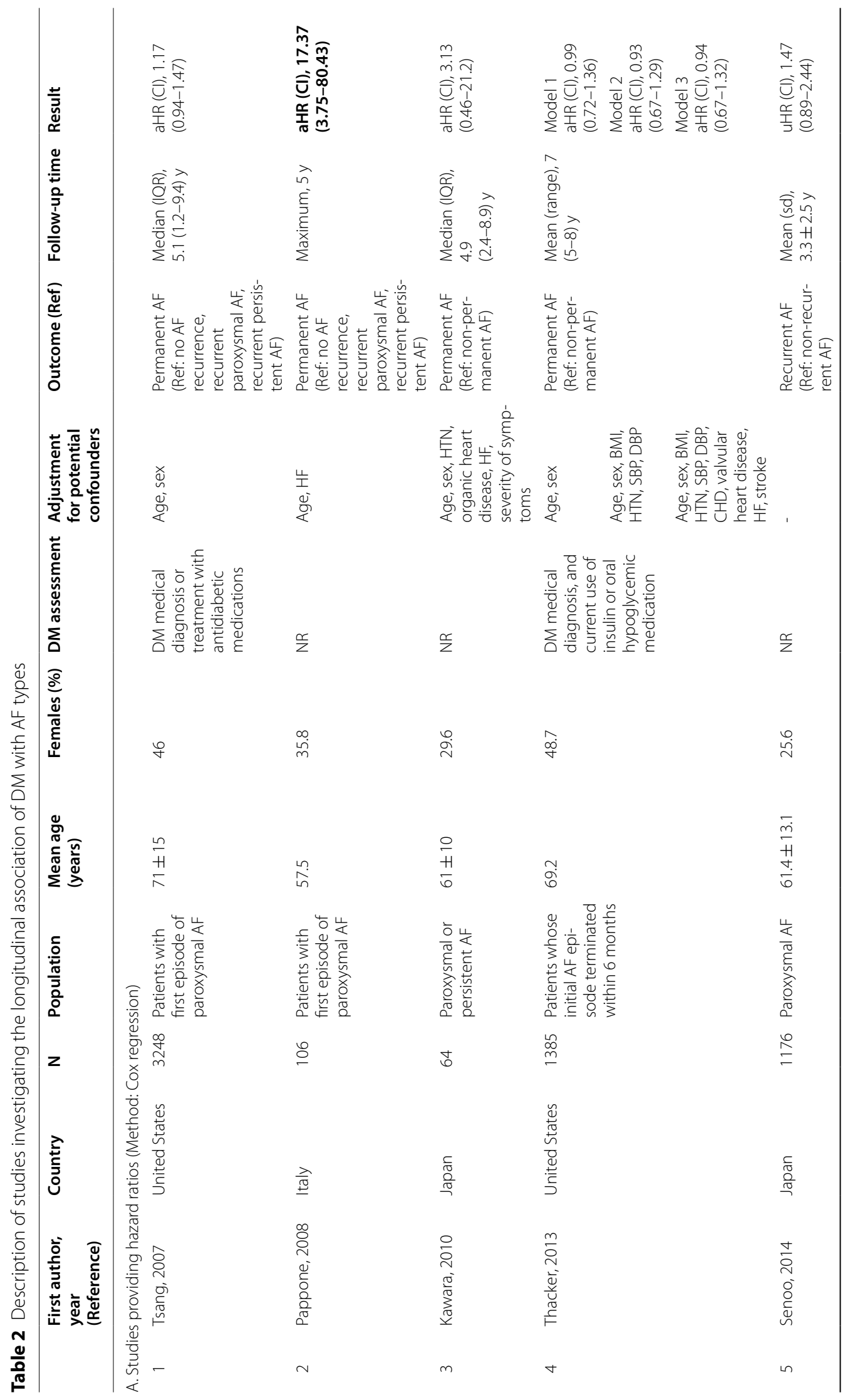




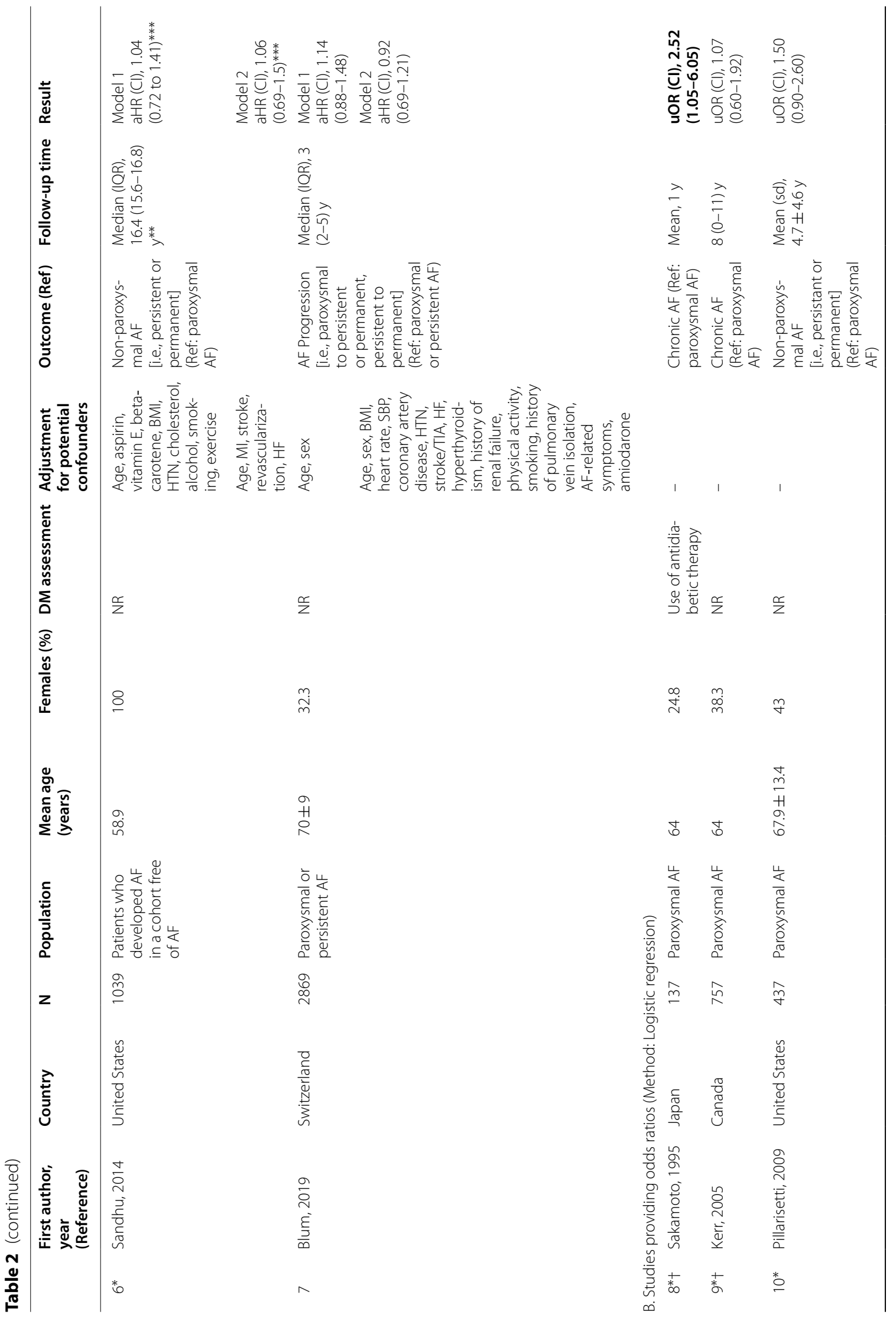




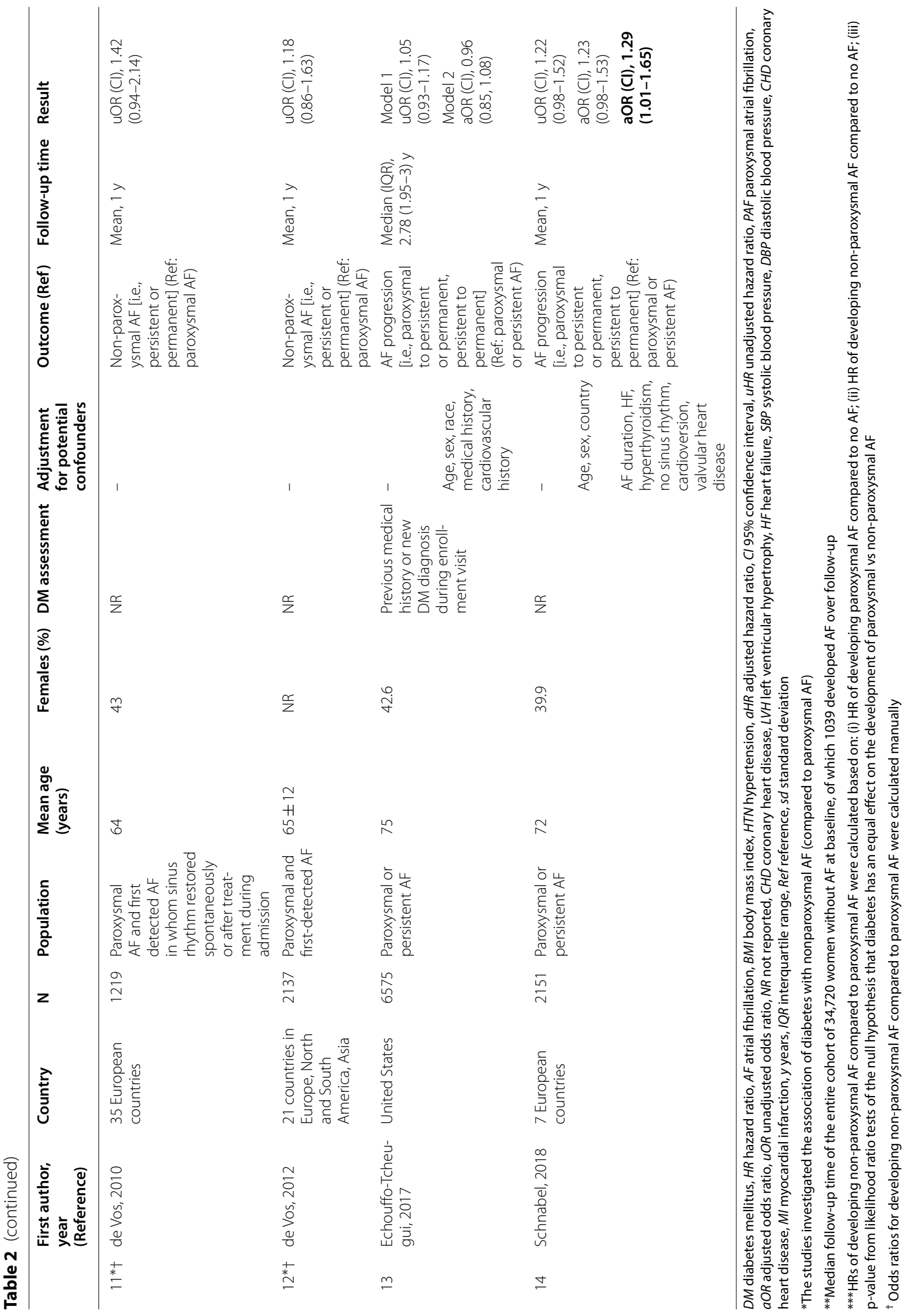




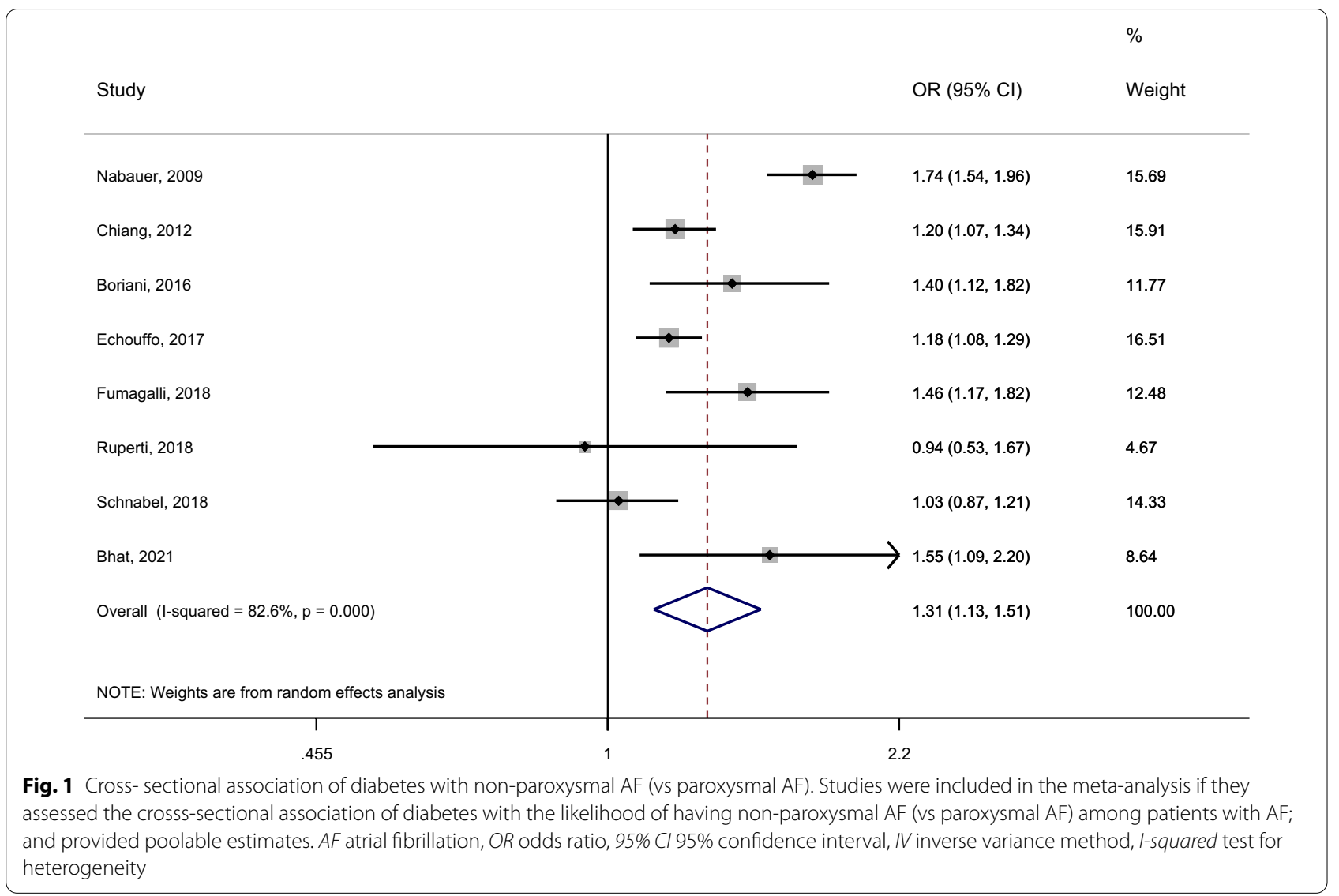

persistent or permanent; persistent to permanent) over the follow-up period [15, 23, 29] (Table 2, Additional file 1: Table S3). Eight studies included patients with paroxysmal AF at baseline; these studies investigated the association of diabetes with the likelihood of developing either "permanent AF" [12, 14], "recurrent AF" [31], "chronic AF", or "non-paroxysmal AF" [7, 26, 32, 33, 35] (Table 2, Additional file 1: Table S3). Of these 8 studies, 2 studies showed an association between diabetes and incident non-paroxysmal AF and permanent AF, respectively (Table 2) [14, 32]. The other 6 studies reported no association between diabetes and AF types (Table 2). We included 5 of 14 longitudinal studies in our meta-analysis; these 5 studies classified AF types into paroxysmal (reference) vs non-paroxysmal AF (Fig. 2). The meta-analysis showed that among patients with paroxysmal AF, diabetes was associated with a 1.32-times increased likelihood of developing non-paroxysmal AF with no heterogeneity (pooled OR [95\% CI]; 1.32 [1.07-1.62]; $\mathrm{I}^{2}=0 \%$ ) (Fig. 2). All studies we included in our meta-analysis provided unadjusted estimates (Table 2, Fig. 2).

\section{Sensitivity analyses}

Results from our meta-analyses of cross-sectional and longitudinal studies did not change substantially after removing studies one by one from the analyses (Figs. 1, 2, Additional file 1: Table S5). In the meta-analysis of cross-sectional studies, the heterogeneity decreased from high $\left(\mathrm{I}^{2}, 82.6 \%\right)$ to moderate $\left(\mathrm{I}^{2}, 44 \%\right)$ after removing the study from Nabauer et al. [13] (Additional file 1: Table S5). In the meta-analysis of longitudinal studies, results remained consistent after performing subgroup analyses based on follow-up time and also after restricting the analysis to studies that defined AF types in accordance with guidelines (Additional file 1: Table S6).

\section{Quality assessment}

Of the 20 included studies, 12 (60\%) were poor quality, 1 (5\%) fair quality, and 7 (35\%) good quality. Out of 9 stars, 6 studies scored 8 stars; 6 scored 6; 4 scored 5; and 4 scored 4 . We present study quality assessment scores in Additional file 1: Table S7. 


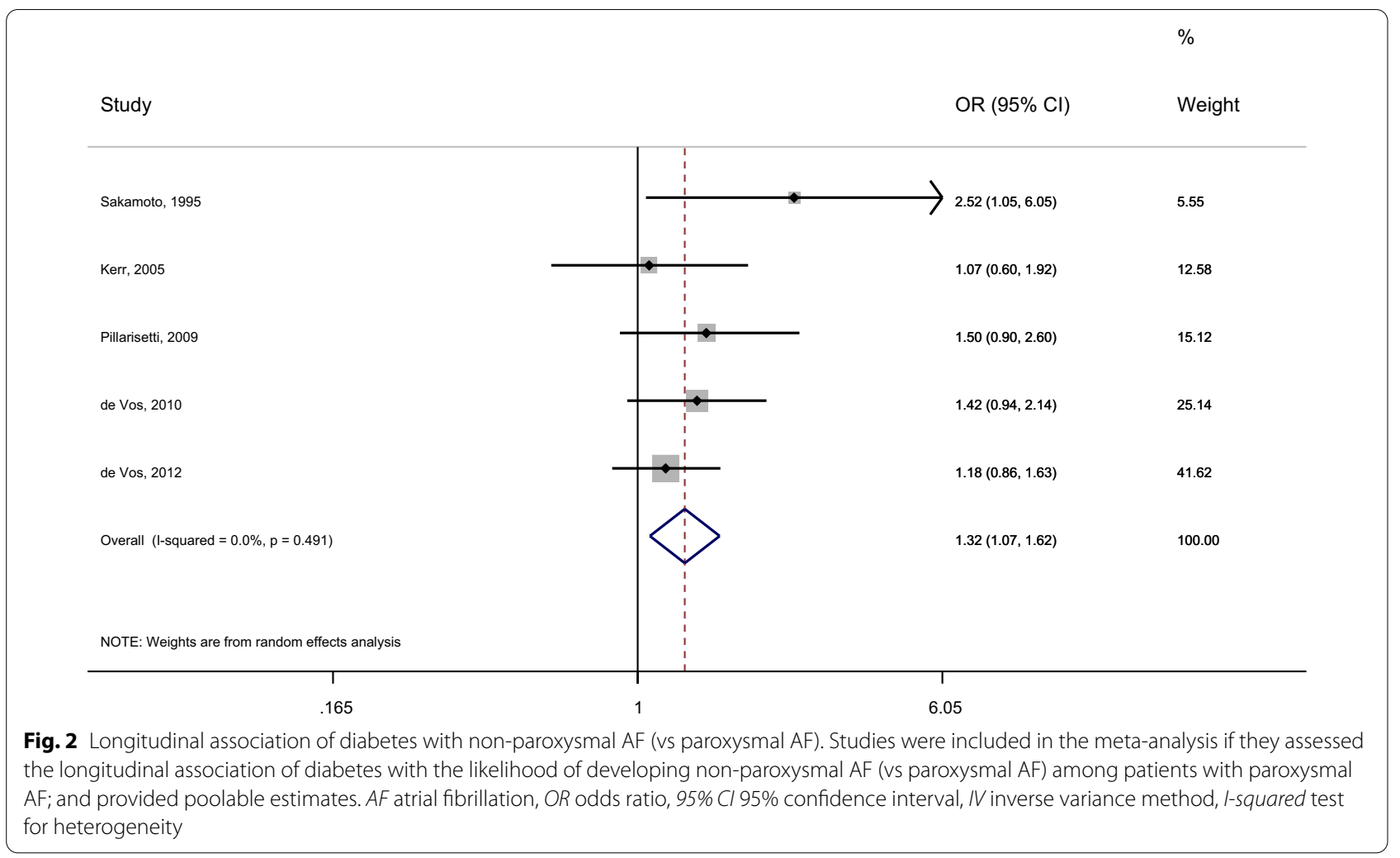

\section{Assessment of publication bias}

We show funnel plots for the cross-sectional and longitudinal association of diabetes with non-paroxysmal AF (vs paroxysmal AF) in Additional file 1: Fig. S2. The Egger`s regression test did not show significant funnel plot asymmetry (p-values 0.8 and 0.2 , respectively). Hence, even though it should be noted that a maximum of eight studies were included, there was no evidence of publication bias.

\section{Discussion \\ Main findings}

To the best of our knowledge, ours is the first systematic review which summarizes the literature regarding the association of diabetes with AF types in humans. Furthermore, this is the first meta-analysis which evaluates the likelihood of having non-paroxysmal AF rather than paroxysmal AF among patients with and without diabetes. We included various studies with relatively large sample sizes of participants with a wide range of ages from different parts of the world. The quality of studies spanned poor (60\%), fair (5\%), and good (35\%). The metaanalysis of cross-sectional studies suggested that patients with AF and diabetes have a 1.31-times higher likelihood of non-paroxysmal AF when compared to those without diabetes. Our meta-analysis of longitudinal studies suggested that among patients with paroxysmal AF, the presence of diabetes is associated with 1.32-times higher likelihood of progression to non-paroxysmal AF. Additional sensitivity analyses provided similar findings. No evidence of publication bias was observed in included studies. The meta-analysis of cross-sectional studies indicated a high heterogeneity. However, the heterogeneity was reduced $\left(\mathrm{I}^{2}=44 \%\right)$ in the "leave one out analysis" when removing the study from Nabauer et al. [13]. The study from Nabauer et al. was conducted over the time period 2004-2006, and it was the earliest investigation reporting on the cross-sectional association of diabetes with AF types, compared to the other studies. Hence, the result of the "leave-one out analysis" might be explained by the differences in the recruitment periods across studies, which can reflect differences over time in the definitions of AF types and management of patients with AF. No heterogeneity was observed in the meta-analysis of longitudinal studies.

\section{Mechanisms linking diabetes to non-paroxysmal AF}

Several mechanisms linking diabetes to non-paroxysmal AF can be proposed, including progression of atrial remodelling, worsening atrial cardiomyopathy, and development of cardiometabolic diseases [6]. Diabetes can lead to left ventricular hypertrophy and dysfunction, 
which in turn increase atrial afterload and promote left atrial dilation. These conditions further foster the maintenance and recurrence of AF [26, 38]. Furthermore, diabetes is accompanied by an inflammatory state and oxidative stress, which are characterized by elevated levels of C-reactive protein, tumor necrosis factor-alpha, interleukin-6, and reactive oxygen species [9, 39]. Inflammation and oxidative stress are key mediators of proarrhythmic atrial remodeling among patients with diabetes $[9,39]$. Diabetes is also associated with atrial fibrosis, which can promote gene expression that enhances the proliferation of fibroblasts and increases extra-cellular matrix secreting function [40]. This underlies the progression of AF to sustained forms by creating a long-term positive feedback loop-the so-called "AF begets AF" hypothesis [40]. Lastly, diabetes increases the risk of cardiometabolic diseases, such as coronary artery disease, hypertension, heart failure, and chronic kidney disease, which further increase susceptibility to non-paroxysmal AF [7, 29, 40-42].

\section{Lessons learned and future perspectives}

Our study highlights the need for improving the quality of research about diabetes and AF types, while also proposing new research directions for the future. First, the studies we included in our systematic review used different categorizations of AF types, such as "paroxysmal" vs "non-paroxysmal" AF or "non-permanent" (i.e., paroxysmal or persistent) vs "permanent" AF. Although classification as paroxysmal or non-paroxysmal reflects the pathophysiology of AF, classification as non-permanent or permanent mainly reflects the therapeutic attitude of the patient and physician. For instance, older patients and those who have comorbidities are more likely to be considered as having permanent AF. Future studies aiming to provide mechanistic insights about diabetes and AF types should thus focus on the classification of AF types as paroxysmal and non-paroxysmal AF [6].

Second, longitudinal studies included in our systematic review focused on several transitions of AF types. One study followed-up patients without AF and classified them based on AF development (i.e., no AF, paroxysmal AF, non-paroxysmal AF) [25]. Some studies followed-up patients with paroxysmal AF and classified them based on recurrence [12, 14, 31]; and other studies followed-up patients with paroxysmal or persistent $\mathrm{AF}$ and classified them based on AF progression (i.e., paroxysmal to persistent or permanent; persistent to permanent) $[15,23$, 29]. In general, AF was documented by medical records, clinical visits, ECG recordings and/or Holter monitoring, which were performed at baseline and over a limited number of follow-up visits. Indeed, AF is a dynamic disease. Over time, patients without AF can progress to paroxysmal or non-paroxysmal AF; and patients with paroxysmal AF can also progress to non-paroxysmal AF [33, 43]. Since evaluation requires a patient's repeated long-term rhythm monitoring, evaluating AF types and progression can thus be challenging. In particular, the limited number and small frequency of AF evaluations may impede the detection of paroxysmal AF. This may further hamper the comparison between AF types. Future studies with long-term follow-up need to comprehensively account for the progressive nature of $\mathrm{AF}$ and evaluate the role of diabetes in these dynamic processes.

Third, all studies in our systematic review that investigated the longitudinal association of diabetes with AF progression only evaluated diabetes status at baseline. Future studies should account for the potential development of incident diabetes over time and also apply Cox models with time-varying covariates.

Fourth, most studies included in our systematic review did not describe how they defined diabetes. Some studies also used outdated terminology for AF types (e.g., "chronic AF") [32, 33]. Future investigations thus need to define diabetes diagnosis in accordance with recommendations and use the most recent terminology for and definitions of AF types [6,44].

Fifth, we observed different levels of adjustments across studies in the systematic review; some studies did not adjust for potential confounders, some adjusted for age and sex, and others adjusted for cardiovascular risk factors and/or cardiometabolic diseases. In general, effect estimates attenuated after adjusting for cardiovascular risk factors (e.g., body mass index, hypertension, systolic and diastolic blood pressure) and/or cardiometabolic diseases (e.g., coronary heart disease, revascularization, valvular heart disease, heart failure, stroke, renal failure, and thyroid dysfunction). This can be explained by the fact that some of these factors can be on the path linking diabetes to AF types. Future studies are warranted to clarify whether these factors have a mediating or confounding role in the association of diabetes with AF types. The studies that were eligible to be included in the meta-analyses of cross-sectional and longitudinal studies were mostly or completely unadjusted for potential confounders. In particular, adjusting for age and sex is crucial when investigating the association of diabetes with AF types, and future studies need to account for these factors. Additional adjustments for confounders, including alcohol, smoking, physical activity, education, medications, need to be performed accordingly.

Sixth, most studies included in the systematic review and meta-analysis did not take into account AF duration, which represents an important aspect in the examination of AF pattern. Hypothetically, underlying cardiovascular risk factors, such as diabetes, can be associated with a 
longer duration of atrial remodelling. In turn, AF duration can be associated with an increased likelihood of non-paroxysmal AF [45]. However, in one of the studies of our systematic review, the association between diabetes and non-paroxysmal AF became stronger and statistically significant after adjustment for AF duration, among other factors [15]. Future studies are needed to provide well characterized data on AF duration.

Although the results of our cross-sectional and longitudinal analyses were consistent, we can not exclude the possibility of residual or unmeasured confounding, due to the observational character of our study. Future studies (e.g., Mendelian Randomization) need to establish if the association of diabetes with non-paroxysmal AF is causal.

Finally, the increased risk of bias in our systematic review and meta-analysis was mainly explained by concerns related to definitions of AF types and adjustments for potential confounders. Our study highlights the need for improving these aspects and the overall quality of evidence in the future.

\section{Areas of limited evidence and future perspectives}

Diabetes and non-paroxysmal AF We were able to examine two specific research questions in our meta-analyses: (1) "Among patients with AF, is the presence of diabetes associated with an increased likelihood of having nonparoxysmal AF rather than paroxysmal AF?" (2) and "Among patients with paroxysmal AF, is the presence of diabetes associated with an increased likelihood of progression to non-paroxysmal AF?" Another important research question would be: "Among patients without AF, is the presence of diabetes associated with an increased risk of developing non-paroxysmal AF rather than paroxysmal AF?" Since only one study investigated this research question, we could not perform a meta-analysis on this matter [25]. Future studies are needed to evaluate the association of diabetes with paroxysmal AF (vs no $\mathrm{AF}$ ) and of diabetes with non-paroxysmal AF (vs no AF), and assess whether there are significant differences.

Glycemic control and non-paroxysmal AF Given the scarcity of evidence in the field, we could not perform a systematic review on the association between glycemic control and AF types. So far, increasing concentrations of glycated hemoglobin (HbA1c) and long term glycemic variability have been prospectively associated with an increased risk of incident AF [46-48]. In accordance, we hypothesize that patients with diabetes and inadequate glycemic control are more likely to have non-paroxysmal AF rather than paroxysmal AF. This hypothesis was also confirmed in a study reporting a positive linear association of HbA1c levels with non-paroxysmal AF [25]. Future studies may need to evaluate whether it is useful to integrate diabetes and measures of glycemic control in risk scores for predicting the risk of incident non-paroxysmal AF among those without AF or with paroxysmal AF, respectively.

SGLT2 inhibitors and non-paroxysmal AF Recent studies have shown that sodium dependent glucose cotransporter 2 inhibitors have beneficial effects reducing the risk of incident $\mathrm{AF}$ and improving $\mathrm{AF}$ prognosis [49-52]. It can thus be assumed that this therapeutic option could also reduce the risk of non-paroxysmal AF. However, further studies are warranted to confirm this assumption.

\section{Conclusions and implications}

Our meta-analysis findings suggest that diabetes is associated with increased likelihood of non-paroxysmal AF rather than paroxysmal AF. Our systematic review provides a comprehensive summary of evidence about the association of diabetes with AF types. These insights allowed us to identify current limitations and propose new directions for the improvement of future research about diabetes and AF types. Specifically, future studies should be based on classifying AF types into paroxysmal vs non-paroxysmal AF, properly adjusting for confounders, accounting for incident diabetes using Cox models with time-varying covariates, as well as using standard definitions for diabetes and AF types in accordance with existing recommendations. Further high quality studies are needed to replicate our findings, examine causality, elucidate the exact mechanisms linking diabetes to nonparoxysmal AF, evaluate the potential value of diabetes in predicting non-paroxysmal AF, and assess the role of glycemic control and antidiabetic medications on AF types.

Our systematic review and meta-analysis provides insights into the pathophysiology of AF. Our findings suggest that patients with diabetes might need more attentive care than those without diabetes, in order to halt progression of AF burden and prevent adverse cardiovascular events. Our data can also imply that the substrate to ablate might be more complex in patients with diabetes than in those without diabetes. Future strategies can foster prevention of non-paroxysmal AF by close monitoring of patients at high risk, increasing patient awareness, and involving patients in treatment plans. In addition, future studies could explore markers that may be used in the clinical setting to identify patients with diabetes at increased risk of AF progression, thus improving personalized care.

\footnotetext{
Abbreviations

AF: Atrial fibrillation; HbA1c: Glycated hemoglobin; HR: Hazard ratio; OR: Odds ratio; 95\% Cl: 95\% Confidence intervals; NOS: Newcastle-Ottawa Scale.
} 


\section{Supplementary Information}

The online version contains supplementary material available at https://doi. org/10.1186/s12933-021-01423-2.

\begin{abstract}
Additional file 1. Supplemental Material. Appendix A. Supplemental information on search strategy. Appendix B. Adapted scale from the Newcastle-Ottawa quality assessment scale for cohort studies. Fig. S1. Flowchart for study inclusion. Fig. S2. Funnel plots on the cross-sectional and longitudinal association of diabetes with non-paroxysmal AF (vs paroxysmal AF). Table S1. Classification of AF types, based on the presentation, duration, and spontaneous termination of AF episodes. Table S2. Recruitment setting. Table S3. AF definitions across studies. Table S4. AF monitoring in studies investigating the longitudinal association of diabetes with AF types. Table S5. "Leave one out" sensitivity analysis. Table S6. Sensitivity analyses in the meta-analysis of longitudinal studies investigating the association of diabetes with non-paroxysmal AF (vs paroxysmal AF). Table S7. Quality assessment scale.
\end{abstract}

\section{Acknowledgements}

We thank Kristin Marie Bivens (Institute of Social and Preventive Medicine [ISPM]) for her editorial assistance.

\section{Authors' contributions}

All authors listed have made a substantial, direct, and intellectual contribution to the work, as well as approved it for publication. All authors read and approved the final manuscript.

\section{Funding}

None.

\section{Availability of data and materials}

Data sharing is not applicable to this article as no datasets were generated or analyzed during the current study.

\section{Declarations}

\section{Ethics approval and consent to participate}

Not applicable.

\section{Consent for publication}

Not applicable.

\section{Competing interests}

The authors have no disclosures to report.

\begin{abstract}
Author details
${ }^{1}$ Institute of Social and Preventive Medicine (ISPM), University of Bern, Mittelstrasse 43, 3012 Bern, Switzerland. ' Department of Cardiology, Inselspital, Bern University Hospital, University of Bern, Bern, Switzerland. ${ }^{3}$ Department of Endocrinology, Gateshead Health NHS Foundation Trust, Gateshead, UK. ${ }^{4}$ Translational and Clinical Research Institute, Newcastle University, Newcastle upon Tyne, UK. ${ }^{5}$ Public Health and Primary Care Library, University Library of Bern, University of Bern, Bern, Switzerland.
\end{abstract}

Received: 28 September 2021 Accepted: 21 November 2021 Published online: 07 December 2021

\section{References}

1. Wolf PA, Abbott RD, Kannel WB. Atrial fibrillation as an independent risk factor for stroke: the Framingham Study. Stroke. 1991;22(8):983-8.

2. Benjamin EJ, Wolf PA, D'Agostino RB, Silbershatz H, Kannel WB, Levy D. Impact of atrial fibrillation on the risk of death: the Framingham Heart Study. Circulation. 1998;98(10):946-52.

3. Kim MH, Johnston SS, Chu BC, Dalal MR, Schulman KL. Estimation of total incremental health care costs in patients with atrial fibrillation in the United States. Circ Cardiovasc Qual Outcomes. 2011;4(3):313-20.
4. Piccini JP, Hammill BG, Sinner MF, Jensen PN, Hernandez AF, Heckbert SR, et al. Incidence and prevalence of atrial fibrillation and associated mortality among Medicare beneficiaries, 1993-2007. Circ Cardiovasc Qual Outcomes. 2012;5(1):85-93.

5. Camm AJ, Kirchhof P, Lip GY, Schotten U, Savelieva I, Ernst S, et al. Guidelines for the management of atrial fibrillation: the Task Force for the Management of Atrial Fibrillation of the European Society of Cardiology (ESC). Eur Heart J. 2010;31(19):2369-429.

6. Hindricks G, Potpara T, Dagres N, Arbelo E, Bax JJ, Blomström-Lundqvist C, et al. 2020 ESC Guidelines for the diagnosis and management of atrial fibrillation developed in collaboration with the European Association for Cardio-Thoracic Surgery (EACTS). Eur Heart J. 2021;42(5):373-498.

7. de Vos CB, Pisters R, Nieuwlaat R, Prins MH, Tieleman RG, Coelen RJ, et al. Progression from paroxysmal to persistent atrial fibrillation clinical correlates and prognosis. J Am Coll Cardiol. 2010;55(8):725-31.

8. Ganesan AN, Chew DP, Hartshorne T, Selvanayagam JB, Aylward PE, Sanders $P$, et al. The impact of atrial fibrillation type on the risk of thromboembolism, mortality, and bleeding: a systematic review and meta-analysis. Eur Heart J. 2016;37(20):1591-602.

9. Wang A, Green JB, Halperin JL, Piccini JP Sr. Atrial fibrillation and diabetes mellitus: JACC review topic of the week. J Am Coll Cardiol. 2019;74(8):1107-15.

10. Huxley RR, Filion KB, Konety S, Alonso A. Meta-analysis of cohort and case-control studies of type 2 diabetes mellitus and risk of atrial fibrillation. Am J Cardiol. 2011;108(1):56-62.

11. Goudis CA, Korantzopoulos P, Ntalas IV, Kallergis EM, Liu T, Ketikoglou DG. Diabetes mellitus and atrial fibrillation: pathophysiological mechanisms and potential upstream therapies. Int J Cardiol. 2015;184:617-22.

12. Tsang TS, Barnes ME, Miyasaka Y, Cha SS, Bailey KR, Verzosa GC, et al. Obesity as a risk factor for the progression of paroxysmal to permanent atrial fibrillation: a longitudinal cohort study of 21 years. Eur Heart J. 2008;29(18):2227-33.

13. Nabauer M, Gerth A, Limbourg T, Schneider S, Oeff M, Kirchhof P, et al. The registry of the German competence NETwork on atrial fibrillation: patient characteristics and initial management. Europace. 2009;11(4):423-34.

14. Pappone C, Radinovic A, Manguso F, Vicedomini G, Ciconte G, Sacchi S, et al. Atrial fibrillation progression and management: a 5-year prospective follow-up study. Heart Rhythm. 2008:5(11):1501-7.

15. Schnabel RB, Pecen L, Engler D, Lucerna M, Sellal JM, Ojeda FM, et al. Atrial fibrillation patterns are associated with arrhythmia progression and clinical outcomes. Heart. 2018;104(19):1608-14.

16. Ruperti Repilado FJ, Doerig L, Blum S, Aeschbacher S, Krisai P, Ammann P, et al. Prevalence and predictors of atrial fibrillation type among individuals with recent onset of atrial fibrillation. Swiss Med Wkly. 2018;148:14652.

17. Muka T, Glisic M, Milic J, Verhoog S, Bohlius J, Bramer W, et al. A 24-step guide on how to design, conduct, and successfully publish a systematic review and meta-analysis in medical research. Eur J Epidemiol. 2020;35(1):49-60

18. Moher D, Liberati A, Tetzlaff J, Altman DG. Preferred reporting items for systematic reviews and meta-analyses: the PRISMA statement. PLoS Med. 2009;6(7):e1000097.

19. GA Wells BS, D O'Connell, J Peterson, VWelch, M Losos, P Tugwell. The Newcastle-Ottawa Scale (NOS) for assessing the quality of nonrandomised studies in meta-analyses 2000. [Available from: http://www.ohri. $\mathrm{ca} /$ programs/clinical_epidemiology/oxford.asp.

20. Stang A. Critical evaluation of the Newcastle-Ottawa scale for the assessment of the quality of nonrandomized studies in meta-analyses. Eur J Epidemiol. 2010;25(9):603-5.

21. Der Simonian R, Laird N. Metaanalysis in clinical trials. Control Clin Trials. 1986;7:177-88.

22. Egger M, Davey Smith G, Schneider M, Minder C. Bias in meta-analysis detected by a simple, graphical test. BMJ (Clinical research ed). 1997;315(7109):629-34

23. Echouffo-Tcheugui JB, Shrader P, Thomas L, Gersh BJ, Kowey PR, Mahaffey KW, et al. Care patterns and outcomes in atrial fibrillation patients with and without diabetes: ORBIT-AF registry. J Am Coll Cardiol. 2017;70(11):1325-35

24. Thacker EL, McKnight B, Psaty BM, Longstreth WT Jr, Dublin S, Jensen PN, et al. Association of body mass index, diabetes, hypertension, and blood pressure levels with risk of permanent atrial fibrillation. J Gen Intern Med. 2013:28(2):247-53. 
25. Sandhu RK, Conen D, Tedrow UB, Fitzgerald KC, Pradhan AD, Ridker PM, et al. Predisposing factors associated with development of persistent compared with paroxysmal atrial fibrillation. J Am Heart Assoc. 2014;3(3).

26. Pillarisetti J, Patel A, Boc K, Bommana S, Sawers Y, Vanga S, et al. Evolution of paroxysmal atrial fibrillation to persistent or permanent atrial fibrillation: predictors of progression. J Atrial Fibrillation. 2009;1 (7):388-94.

27. Boriani G, Laroche C, Diemberger I, Fantecchi E, Popescu MI, Rasmussen $L \mathrm{H}$, et al. 'Real-world'management and outcomes of patients with paroxysmal vs. non-paroxysmal atrial fibrillation in Europe: the EURObservational Research Programme-Atrial Fibrillation (EORP-AF) General Pilot Registry. Europace. 2016;18(5):648-57.

28. Fumagalli S, Said SA, Laroche C, Gabbai D, Boni S, Marchionni N, et al. Management and prognosis of atrial fibrillation in diabetic patients: an EORP-AF General Pilot Registry report. Eur Heart J Cardiovasc Pharmacother. 2018;4(3):172-9.

29. Blum S, Aeschbacher S, Meyre P, Zwimpfer L, Reichlin T, Beer JH, et al. Incidence and predictors of atrial fibrillation progression. J Am Heart Assoc. 2019;8(20):e012554.

30. Kawara T, Narumi J, Hirao K, Kasuya K, Kawabata M, Tojo N, et al. Symptoms of atrial fibrillation in patients with and without subsequent permanent atrial fibrillation based on a retrospective questionnaire survey. Int Heart J. 2010;51(4):242-6.

31. Senoo K, Suzuki S, Otsuka T, Sagara K, Matsuno S, Kano H, et al. Progression to the persistent form in asymptomatic paroxysmal atrial fibrillation. Circ J. 2014;78(5):1121-6.

32. Sakamoto H, Okamoto IE, Imataka K, leki K, Fujui J. Prediction of early development of chronic nonrheumatic atrial fibrillation. Jpn Heart J. 1995;36(2):191-9.

33. Kerr CR, Humphries KH, Talajic M, Klein GJ, Connolly SJ, Green M, et al. Progression to chronic atrial fibrillation after the initial diagnosis of paroxysmal atrial fibrillation: results from the Canadian Registry of Atrial Fibrillation. Am Heart J. 2005;149(3):489-96.

34. Bhat A, Chen HHL, Khanna S, Gan GCH, Abhayaratna WP, Nunes MCP, et al. Clinical and cardiac structural predictors of atrial fibrillation persistence. Eur J Clin Invest. 2021;51(3):e13395.

35. Vos CBD, Breithardt G, Camm AJ, Dorian P. Progression of atrial fibrillation in the REgistry on Cardiac rhythm disORDers assessing the control of Atrial Fibrillation cohort: clinical correlates and the effect. Elsevier; 2012.

36. Chiang CE, Naditch-Brûlé L, Murin J, Goethals M, Inoue H, O-Neill J, et al. Distribution and risk profile of paroxysmal, persistent, and permanent atrial fibrillation in routine clinical practice insight from the real-life global survey evaluating patients with atrial fibrillation international registry. Circ Arrhythm Electrophysiol. 2012;5(4):632-9.

37. Potpara TS, Stankovic GR, Beleslin BD, Polovina MM, Marinkovic JM, Ostojic MC, et al. A 12-year follow-up study of patients with newly diagnosed lone atrial fibrillation. Implications of arrhythmia progression on prognosis: the Belgrade atrial fibrillation study. Chest. 2012;141(2):339-47.

38. Rutter MK, Parise H, Benjamin EJ, Levy D, Larson MG, Meigs JB, et al. Impact of glucose intolerance and insulin resistance on cardiac structure and function: sex-related differences in the Framingham Heart Study. Circulation. 2003;107(3):448-54.

39. Scridon A, Dobreanu D, Chevalier P, Şerban RC. Inflammation, a link between obesity and atrial fibrillation. Inflamm Res. 2015;64(6):383-93.

40. Nattel S, Guasch E, Savelieva I, Cosio FG, Valverde I, Halperin JL, et al. Early management of atrial fibrillation to prevent cardiovascular complications. Eur Heart J. 2014;35(22):1448-56.

41. Parkash R. Triggers, substrate, and hypertension in atrial fibrillation: how does it all add up? JACC Clin Electrophysiol. 2015;1 (3):174-6.

42. Deng H, Bai Y, Shantsila A, Fauchier L, Potpara TS, Lip GYH. Clinical scores for outcomes of rhythm control or arrhythmia progression in patients with atrial fibrillation: a systematic review. Clin Res Cardiol. 2017;106(10):813-23.

43. Rostagno C, Bacci F, Martelli M, Naldoni A, Bertini G, Gensini G. Clinical course of lone atrial fibrillation since first symptomatic arrhythmic episode. Am J Cardiol. 1995;76(11):837-9.

44. Classification and diagnosis of diabetes: standards of medical care in diabetes-2020. Diabetes care. 2020;43(Suppl 1):S14-s31.

45. Holmqvist F, Kim S, Steinberg BA, Reiffel JA, Mahaffey KW, Gersh BJ, et al. Heart rate is associated with progression of atrial fibrillation, independent of rhythm. Heart. 2015;101(11):894-9.
46. Qi W, Zhang N, Korantzopoulos P, Letsas KP, Cheng M, Di F, et al. Serum glycated hemoglobin level as a predictor of atrial fibrillation: a systematic review with meta-analysis and meta-regression. PLoS ONE. 2017;12(3):e0170955.

47. Gu J, Fan YQ, Zhang JF, Wang CQ. Impact of long-term glycemic variability on development of atrial fibrillation in type 2 diabetic patients. Anatol J Cardiol. 2017;18(6):410-6.

48. Seyed Ahmadi S, Svensson AM, Pivodic A, Rosengren A, Lind M. Risk of atrial fibrillation in persons with type 2 diabetes and the excess risk in relation to glycaemic control and renal function: a Swedish cohort study. Cardiovasc Diabetol. 2020;19(1):9.

49. Okunrintemi V, Mishriky BM, Powell JR, Cummings DM. Sodium-glucose co-transporter-2 inhibitors and atrial fibrillation in the cardiovascular and renal outcome trials. Diabetes Obes Metab. 2021;23(1):276-80.

50. Zelniker TA, Bonaca MP, Furtado RHM, Mosenzon O, Kuder JF, Murphy $\mathrm{SA}$, et al. Effect of dapagliflozin on atrial fibrillation in patients with type 2 diabetes mellitus: insights from the DECLARE-TIMI 58 trial. Circulation. 2020;141(15):1227-34.

51. Böhm M, Slawik J, Brueckmann M, Mattheus M, George JT, Ofstad AP, et al. Efficacy of empagliflozin on heart failure and renal outcomes in patients with atrial fibrillation: data from the EMPA-REG OUTCOME trial. Eur J Heart Fail. 2020;22(1):126-35.

52. Li WJ, Chen XQ, Xu LL, Li YQ, Luo BH. SGLT2 inhibitors and atrial fibrillation in type 2 diabetes: a systematic review with meta-analysis of 16 randomized controlled trials. Cardiovasc Diabetol. 2020;19(1):130.

\section{Publisher's Note}

Springer Nature remains neutral with regard to jurisdictional claims in published maps and institutional affiliations.

Ready to submit your research? Choose BMC and benefit from

- fast, convenient online submission

- thorough peer review by experienced researchers in your field

- rapid publication on acceptance

- support for research data, including large and complex data types

- gold Open Access which fosters wider collaboration and increased citations

- maximum visibility for your research: over $100 \mathrm{M}$ website views per year

At BMC, research is always in progress.

Learn more biomedcentral.com/submissions 